\title{
EFEITOS DE DOSES E FORMAS DE APLICAÇÃO DE REGULADORES DE CRESCIMENTO EM UVAS SEM SEMENTES, CV. BRS CLARA, EM REGIÃO TROPICAL ${ }^{1}$
}

\author{
REGINALDO TEODORO DE SOUZA², JAIR COSTA NACHTIGAL ${ }^{3}$, \\ JOÃO PAULO MORANTE ${ }^{4}$, ANA PAULA DO SANTOS SANTANA ${ }^{5}$
}

RESUMO-O objetivo deste trabalho foi avaliar o efeito do uso dos reguladores vegetais, ácido giberélico e tidiazuron, no tamanho de bagas da cultivar BRS Clara em Região Tropical. Os experimentos foram conduzidos em 2006 e 2007, na Estação Experimental de Viticultura Tropical, em Jales-SP, região noroeste do Estado de São Paulo, em plantas da cv. BRS Clara. Foram testadas diferentes concentrações de GA 3 aplicadas isoladamente, combinadas com Crop Set ou tidiazuron, em aplicações únicas ou sequênciais (2 e 4 vezes). As avaliações foram feitas por ocasião da maturação das uvas, considerando-se a massa fresca dos cachos, dos engaços e das bagas, determinadas por meio de balança analítica; o comprimento e o diâmetro médio das bagas, utilizando-se de paquímetro; e o teor de sólidos solúveis (SS), por meio de refratômetro manual. Os experimentos foram conduzidos em delineamento experimental de blocos casualizados, representados por uma planta, com 15 repetições, no ano de 2006, e 10 repetições, no ano de 2007. Os dados foram submetidos à análise de variância e, para a comparação das médias dos tratamentos, foi utilizado o teste Skott Nott, ao nível de $5 \%$ de probabilidade. Observou-se que o uso dos reguladores promoveu o crescimento das bagas na cultivar BRS Clara em todos os tratamentos, diferindo da testemunha.

Termos para indexação: videira, tamanho de bagas, ácido giberélico, tidiazuron.

\section{EFFECTS OF PLANT GROWTH REGULATORS IN SEEDLESS GRAPE CV. BRS CLARA, IN TROPICAL REGION}

\begin{abstract}
The purpose of this study was to evaluate the effect of the use of plant growth regulators, gibberellic acid and thidiazuron on berries size of BRS Clara in Tropical Region. The experiments were conducted in 2006 and 2007, in the Experimental Station of Tropical Viticulture in Jales, SP, northwestern region in the state of São Paulo. It was tested different concentrations of $\mathrm{GA}_{3}$ applied in isolation, combined with Crop set and thidiazuron, in single or sequential applications ( 2 and 4 times). The assessments were made during the ripening of the grapes, considering the masses of fresh bunch of rachis and berry, determined by analytical balance, the average length and the diameter of the berries, using caliper and the content of soluble solids (TSS), through manual refractometer. The experiments were conducted in a randomized complete block design, represented by a plant, with 15 repetitions in 2006 and 10 repetitions in the 2007 . The data were subjected to analysis of variance and for the comparison of the average number of treatments it was used the Skott Nott test at the level of 5\% probability. It was observed that the use of plant growth regulators promoted the growth of berries in BRS Clara, in all treatments differing of the control.
\end{abstract}

Index terms: vine, size of berries, gibberellic acid, thidiazuron.

\footnotetext{
1(Trabalho 188-09). Recebeido em: 13-08-2009. Aceito para publicação em: 19-01-2010.

${ }^{2}$ Doutor em Agronomia, Embrapa Uva e Vinho - Estação Experimental de Viticultura Tropical, C. P. 241, CEP15700-000, Jales-SP, E-mail: recco@cnpuv.embrapa.br;

${ }^{3}$ Doutor em Agronomia, Embrapa Clima Temperado - Estação Experimental Cascata, C. P. 403, CEP96001-970, Pelotas-RS, E-mail: jair.nachtigal@cpact.embrapa.br;

${ }^{4}$ Graduado em Biologia, Sitio Vista Alegre, Córrego do Coqueiro, Jales-SP. Bolsista CNPq. E-mail: jpaulomorante@yahoo.com.br ${ }^{5}$ Mestranda em Agronomia-UNESP, Ilha Solteira-SP; R. Esperança, 2062, Jales-SP. Bolsista FAPESP. E-mail: apsanta@bol.com.br
} 


\section{INTRODUÇÃO}

A BRS Clara é uma cultivar de uva sem sementes, lançada em 2003 pela Embrapa Uva e Vinho, que apresenta, naturalmente, bagas com cerca de $15 \mathrm{~mm}$ de diâmetro e $20 \mathrm{~mm}$ de comprimento (CAMARGO et al., 2003), enquanto os padrões para comercialização interna ou para exportação, o mínimo desejável seriam bagas com $17 \mathrm{~mm}$ de diâmetro. A aplicação exógena de reguladores vegetais para aumento do tamanho de bagas pode contribuir para a melhoria da qualidade do cacho e facilitar a comercialização de cultivares de uvas sem sementes (NACHTIGAL et al., 2005).

O ácido giberélico $\left(\mathrm{GA}_{3}\right)$ é o regulador vegetal mais amplamente utilizado na viticultura em nível comercial, visando principalmente ao aumento do tamanho das bagas (BOTELHO et al., 2002). Outros reguladores vegetais são testados para o aumento de tamanho de bagas, tais como o produto comercial Crop Set (Improcrop-Kentucky-USA), que é um bioestimulante vegetal composto de extratos de agave (Yucca shidigera) e micronutrientes minerais com ação semelhante às citocininas (SOUZA LEÃO et al., 2005). As citocininas são substâncias derivadas da adenina, as quais causam divisão celular nas plantas, em geral por uma interação com auxinas. $\mathrm{O}$ thidiazuron (TDZ: N-fenil-N-1,2,3-tidiazol-5-tiuréia) é uma citocinina sintética, cuja eficiência sobre o aumento no tamanho de bagas foi relatada por diversos autores (MIELE ET AL., 2000; BOTELHO ET AL., 2002; NACHTIGAL et al., 2005).

A aplicação de ácido giberélico $\left(\mathrm{GA}_{3}\right)$ pode causar diferentes resultados para o aumento do tamanho das bagas devido a condições climáticas, solo, nutrição, porta-enxerto, vigor, produção, época de aplicação, dosagem, pragas e doenças, entre outros. Nachtigal (2007) verificou que a aplicação de ácido giberélico, em duas vezes, sendo a primeira na fase de chumbinho (bagas com diâmetro médio de $5 \mathrm{a} 6 \mathrm{~mm}$ ) e a segunda 8 a 10 dias após, utilizando concentrações de $50 \mathrm{mg} \mathrm{L}^{-1}$, possibilitou a obtenção de bagas com mais de $24 \mathrm{~mm}$ de diâmetro para a variedade BRS Clara em vinhedos comerciais localizados na Serra Gaúcha.

O mesmo não se verificou em regiões de clima tropical, em trabalhos realizados na região noroeste do Estado de São Paulo, município de Jales, onde os melhores resultados com a aplicação de ácido giberélico aplicado isoladamente ou em mistura com tidiazuron proporcionaram aumentos máximos no diâmetro de bagas até $17 \mathrm{~mm}$ com o parcelamento das aplicações limitadas a duas épocas, com intervalo de 7 dias (NACHTIGAL et al., 2005).
Assim, o objetivo deste trabalho foi avaliar o efeito de doses e formas de aplicação dos reguladores vegetais, ácido giberélico e tidiazuron, no tamanho de bagas da cultivar BRS Clara,em Região Tropical.

\section{MATERIAL E MÉTODOS}

Os experimentos foram conduzidos em 2006 e 2007, na Estação Experimental de Viticultura Tropical, em Jales-SP, região noroeste do Estado de São Paulo, em plantas da cv. BRS Clara, enxertadas no ano de 2000 e 2006, sobre o porta-enxerto IAC 572 'Jales', no espaçamento 2,5 x 2,0m, conduzidas em sistema de latada e com irrigação por microaspersão, apresentando em média 26 ramos por planta.

No ano de 2006, cujo ciclo produtivo teve início em 28 de abril, os tratamentos utilizados foram: ausência de aplicações e sem o uso de água e adjuvantes; $50 \mathrm{mg} \mathrm{L}^{-1}$ de $\mathrm{GA}_{3}$ aplicado isolado com uma e duas aplicações); $50 \mathrm{mg} \mathrm{L}^{-1} \mathrm{de}_{\mathrm{GA}_{3}}$ em mistura com 1 e $2 \%$ de Crop Set ${ }^{\circledR}$; e $10 \mathrm{mg} \mathrm{L}^{-1}$ de $\mathrm{GA}_{3}$ em mistura com $5 \mathrm{mg} \mathrm{L}^{-1}$ de tidiazuron (TDZ) com uma e duas aplicações. A primeira aplicação foi realizada em 29 de junho, quando as bagas estavam com 5 a 7 mm de diâmetro, e a segunda aplicação aos cinco dias após a primeira, com 9 a $10 \mathrm{~mm}$ de diâmetro.

No ano de 2007, o ciclo teve início com a poda de produção realizada no dia 14 de julho. Neste ciclo, o experimento constou de 10 tratamentos: ausência de tratamentos $(0,0)$; aplicações isoladas de $\mathrm{GA}_{3} \mathrm{em} \mathrm{mg} \mathrm{L}^{-1}$ (8, quatro vezes), (10, quatro vezes), (50, uma vez + pente em pré-florescimento), (50, duas vezes) (100, uma vez), (100, duas vezes); e aplicações combinadas de $\mathrm{GA}_{3}+\mathrm{TDZ}$, em mg L-1 $(8+1$, quatro vezes), $(10+5$, uma vez $)$ e $(10+5$, duas vezes $)$ respectivamente. A primeira aplicação foi realizada no dia 03 de setembro, quando as bagas tinham de 4 a $5 \mathrm{~mm}$ de diâmetro. Para os tratamentos com duas ou mais aplicações, foram deixados intervalos de 7 dias com 9 a $10 \mathrm{~mm}$ de diâmetro e 5 dias com 7 a 8 mm de diâmetro, respectivamente.

Em todos os experimentos, as aplicações dos reguladores foram feitas via pulverização localizada nos cachos selecionados dentro do mesmo estádio, até o ponto de escorrimento, utilizando-se de adjuvantes siliconados $(0,001 \%)$. Durante a condução dos experimentos, foram feitas as operações de manejo, controle fitossanitário, irrigações, adubações e outras, conforme as exigências da cultura. Os cachos foram colhidos e embalados em sacos plásticos, acondicionados em caixas e transportados imediatamente ao laboratório

As avaliações foram feitas por ocasião da maturação das uvas, considerando-se as massas fres- 
cas do cacho, do engaço e da baga, determinadas por meio de balança analítica; comprimento e diâmetro médio das bagas, utilizando-se de paquímetro; e o sólidos solúveis (SS), com bagas coletadas ao acaso, por meio de refratômetro manual.

Os experimentos foram conduzidos em delineamento experimental de blocos casualizados, representados por uma planta, com 15 repetições, no ano de 2006, e 10 repetições, no ano de 2007. Os dados foram submetidos à análise de variância e, para a comparação das médias dos tratamentos, foi utilizado o teste Skott Nott, ao nível de 5\% de probabilidade.

\section{RESULTADOS E DISCUSSÃO}

Os resultados do experimento, conduzido no ano de 2006, são apresentados na Tabela 1, na qual se observam diferenças estatísticas significativas da aplicação de reguladores vegetais sobre o comprimento e o diâmetro das bagas, bem como sobre a massa fresca do cacho da cultivar BRS Clara em relação ao tratamento-testemunha (sem aplicação).

A variável peso de massa fresca do engaço apresentou diferenças estatísticas significativas entre os tratamentos, sendo que os tratamentos com uma

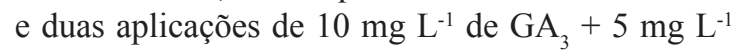
de TDZ apresentaram valores superiores aos demais tratamentos. Quanto à massa fresca das bagas, os tratamentos com a combinação de GA3 e Crop Set $®$ não apresentaram diferenças estatísticas significativas em relação ao tratamento-testemunha, porém apresentaram valores significativamente menores em relação aos demais tratamentos.

Quanto ao teor de sólidos solúveis (SS), todos os tratamentos provocaram redução nos valores de $\mathrm{SS}$, porém os tratamentos com $50 \mathrm{mg} \mathrm{L}^{-1}$ de $\mathrm{GA}_{3}+$ $2 \%$ de Crop Set ${ }^{\circledR}$ e $50 \mathrm{mg} \mathrm{L}^{-1}$ de $\mathrm{GA}_{3}$ com uma única aplicação não apresentaram diferenças estatísticas significativas em relação ao tratamento-testemunha.

Os resultados do experimento, conduzido no ano de 2007, são apresentados na Tabela 2, na qual se observa que os tratamentos utilizados proporcionaram efeitos diferenciados em função das variáveis analisadas. No caso do comprimento das bagas, todos os tratamentos apresentaram diferenças significativas em relação ao tratamento-testemunha, sendo que, de modo geral, os tratamentos com concentrações mais elevadas de reguladores vegetais ou com um número maior de aplicações foram os que proporcionaram os maiores comprimentos de bagas.

O diâmetro das bagas apresentou diferenças estatísticas significativas entre os tratamentos, sendo que os tratamentos com $50 \mathrm{mg} \mathrm{L}^{-1}$ e $100 \mathrm{mg} \mathrm{L}^{-1} \mathrm{de}$ $\mathrm{GA}_{3}$, com uma e duas aplicações, respectivamente, não apresentaram diferenças estatísticas significativas em relação ao tratamento-testemunha, enquanto os cachos do tratamento com $50 \mathrm{mg} \mathrm{L}^{-1}$ de $\mathrm{GA}_{3} \mathrm{e}$ nos quais foi feito o raleio de bagas com pente na pré-floração, apresentaram bagas com diâmetro significativamente inferior ao tratamento-testemunha.

As variáveis peso da massa fresca do cacho, do engaço e das bagas apresentaram comportamento bastante similar quanto ao efeito da aplicação dos diferentes tratamentos, sendo que todos os tratamentos apresentaram valores significativamente superiores ao tratamento-testemunha, exceto o tratamento com aplicação de $50 \mathrm{mg} \mathrm{L}^{-1}$ de $\mathrm{GA}_{3}$ e raleio de bagas com pente na pré-floração, o qual não apresentou diferenças estatísticas significativas em relação ao tratamento-testemunha para estas variáveis.

De forma semelhante ao experimento conduzido durante o ano de 2006, todos os tratamentos com reguladores vegetais provocaram reduções estatisticamente significativas nos teores de SS em relação ao tratamento-testemunha, com exceção do tratamento com aplicação de $50 \mathrm{mg} \mathrm{L}^{-1}$ de $\mathrm{GA}_{3}$ e raleio de bagas com pente na pré-floração, o qual apresentou valores de SS semelhantes ao tratamento-testemunha.

Fazendo-se uma análise conjunta dos resultados obtidos nos dois experimentos, mesmo que tenham sido conduzidos em safras diferentes (2006 e 2007) e com alguns tratamentos também distintos, pode-se verificar que o raleio de bagas para a melhoria da qualidade das bagas e do cacho com o uso de pente ou escova, como o recomendado por diversos autores, entre os quais Leão e Maia (1998), Leão e Possídio (2001) e Pires e Martins (2003), para as cultivares Itália, Rubi, Benitaka e Brasil, não deve ser realizado para a cultivar BRS Clara, uma vez que tal prática provoca redução no diâmetro e na massa das bagas, bem como na massa fresca dos cachos.

Analisando-se o efeito dos reguladores vegetais no diâmetro das bagas, que é uma característica importante no caso das uvas sem sementes, uma vez que é necessário um diâmetro mínimo de $17 \mathrm{~mm}$ para a exportação de uvas apirênicas, verifica-se que o tratamento com $10 \mathrm{mg} \mathrm{L}^{-1} \mathrm{de} \mathrm{GA}_{3}+5 \mathrm{mg} \mathrm{L}^{-1} \mathrm{de}$ TDZ, aplicados duas vezes durante o ciclo da cultura, permite a obtenção de bagos com diâmetro superior a $17 \mathrm{~mm}$. Os valores mais elevados de diâmetro das bagas $(18,15 \mathrm{~mm})$ foram obtidos utilizando-se quatro aplicações de $8 \mathrm{mg} \mathrm{L}^{-1}$ de $\mathrm{GA}_{3}+1 \mathrm{mg} \mathrm{L}^{-1}$ de TDZ, porém houve uma redução significativa no teor de sólidos solúveis, evidenciando o efeito no retardamento da maturação das uvas pela ação 
dos reguladores vegetais, principalmente das citocininas, o que, segundo Metivier (1986), se deve à capacidade de retardar a degradação de proteínas e o desaparecimento da clorofila. Todavia, este efeito muitas vezes pode ser eliminado ou amenizado retardando-se a colheita em uma ou duas semanas (FEITOSA et al., 2002). Também é importante salientar que, mesmo tendo-se colhido todos os tratamentos ao mesmo tempo, o valor de $15,4^{\circ}$ Brix obtido nas uvas no tratamento com $8 \mathrm{mg} \mathrm{L}^{-1}$ de $\mathrm{GA}_{3}+1 \mathrm{mg} \mathrm{L}^{-1}$ de TDZ ainda é superior ao valor de mínimo estabelecido para a comercialização de uvas finas de mesa que é de $14^{\circ}$ Brix.

Em regiões de clima tropical, onde as elevadas temperaturas do ar, associadas às práticas de manejo adequadas, proporcionam uma redução no ciclo da videira, o diâmetro das bagas é menor do que em regiões de clima temperado. A diferença de comportamento da cultivar BRS Clara em função das regiões pode ser comprovada comparando-se os valores de diâmetro de bagos citados por Nachtigal (2007), que obteve bagas com até $24 \mathrm{~mm}$ de diâmetro nas condições do Rio Grande do Sul.

Outro aspecto importante associado ao aumento do diâmetro das bagas é com relação aos efeitos sobre a produção da área. Comparando-se a massa fresca dos cachos nos tratamentos-testemunha, nas duas safras, verifica-se que, na safra de 2006, os cachos eram naturalmente maiores $(504,96 \mathrm{~g})$ do que os da safra de 2007 (355,9 g), porém com o aumento no diâmetro das bagas proporcionado pelos melhores tratamentos (duas aplicações de $10 \mathrm{mg} \mathrm{L}^{-1}$ de $\mathrm{GA}_{3}+5 \mathrm{mg} \mathrm{L}^{-1}$ de TDZ e quatro aplicações de $8 \mathrm{mg} \mathrm{L}^{-1} \mathrm{de}_{\mathrm{GA}_{3}}+1 \mathrm{mg} \mathrm{L}^{-1}$ de TDZ, com 1,59mm em 2006 e 2,41 mm em 2007, respectivamente) houve aumento na massa fresca dos cachos de $77,9 \%$, em 2006, e de 69\%, em 2007, em relação à massa dos cachos dos tratamentos-testemunha. Esse aumento no tamanho dos cachos pelo uso dos reguladores vegetais, associado ao aumento do valor em função da melhor qualidade das bagas (bagas com maior diâmetro), com certeza possibilita maior rentabilidade ao produtor de uvas da cv. BRS Clara.

Embora Souza Leão et al. (2005) tenham observado diferenças significativas em relação ao comprimento e diâmetro de bagos na cultivar Thompson Seedless com a combinação de Crop Set $\AA$ e ácido giberélico, as concentrações utilizadas no presente trabalho, na cultivar BRS Clara em conjunto com o ácido giberélico, apresentaram resultados inferiores ao tratamento somente com ácido giberélico, para as mesmas variáveis. Já a combinação de ácido giberélico com TDZ mostrou resultados satisfatórios, uma vez que o efeito da aplicação em conjunto destes dois reguladores vegetais mostrou-se superior à aplicação isolada de ácido giberélico nas variáveis associadas ao tamanho dos cachos e das bagas, embora provoque redução no teor de sólidos solúveis totais, como pode ser visualizado nos tratamentos com $8 \mathrm{mg} \mathrm{L}^{-1} \mathrm{de}$ $\mathrm{GA}_{3}$ e $8 \mathrm{mg} \mathrm{L}^{-1}$ de $\mathrm{GA}_{3}+1 \mathrm{mg} \mathrm{L}{ }^{-1}$ de TDZ, em 2007. Este efeito sinérgico do ácido giberélico e do TDZ também é mencionado por Botelho et al. (2004) para a cultivar Niágara Rosada. Segundo Mitivier (1986), o tidiazuron promove aumento do tamanho das bagas por induzir a divisão celular, além de interagir com o ácido giberélico no aumento do tamanho das células.

Quanto ao aumento do número de aplicações, não foram observadas diferenças significativas em alguns tratamentos, porém, em outros, os resultados obtidos com duas aplicações foram melhores do que os obtidos com apenas uma aplicação nas variáveis associadas ao tamanho dos cachos e das bagas. No caso dos sólidos solúveis, de modo geral, houve redução nos teores quando se realizou um número maior de aplicações dos reguladores vegetais. Em virtude dos maiores diâmetro e massa fresca das bagas obtidos com o tratamento com quatro aplicações de 8 $\mathrm{mg} \mathrm{L}^{-1}$ de $\mathrm{GA}_{3}+1 \mathrm{mg} \mathrm{L}^{-1}$ de TDZ, faz-se necessária a realização de novos ensaios com parcelamentos e intervalos de aplicações de reguladores vegetais para o aumento do tamanho de bagas em regiões tropicais.

É importante salientar que o tidiazuron não é registrado para a cultura da videira no Brasil, não podendo ser recomendado para o uso comercial (BOTELHO et al., 2004). Entretanto, a realização de trabalhos experimentais pode fornecer subsídios técnicos para o seu registro, uma vez que o ácido giberélico, isoladamente, pode não permitir a obtenção de uvas sem sementes com a qualidade exigida pelo mercado consumidor, principalmente o mercado internacional. 
TABELA 1 - Influência dos reguladores vegetais sobre a massa fresca dos cachos, dos engaços e das bagas, comprimento e diâmetro das bagas e teor de sólidos solúveis totais da cv. BRS Clara. Jales, 2006.

\begin{tabular}{|c|c|c|c|c|c|c|c|}
\hline \multirow{2}{*}{$\begin{array}{l}\text { Tratamento } \\
\text { GA3 }+\ldots .\end{array}$} & \multirow{2}{*}{$\begin{array}{l}\mathrm{N}^{\circ} \mathrm{de} \\
\text { Aplic. }\end{array}$} & \multirow{2}{*}{$\begin{array}{c}\text { Comprim. } \\
\text { de baga }\end{array}$} & \multirow{2}{*}{$\begin{array}{c}\text { Diâmetro } \\
\text { de baga }\end{array}$} & \multirow{2}{*}{ SST } & \multicolumn{3}{|c|}{ Massa fresca } \\
\hline & & & & & Cacho & Engaço & Bagas \\
\hline$\left(\mathrm{mg} \mathrm{L}^{-1}\right)$ & & \multicolumn{2}{|c|}{$(\mathrm{mm})$} & $\left({ }^{\circ}\right.$ Brix $)$ & & (g) & \\
\hline Testemunha & 0 & $20,61 \mathrm{a}$ & $15,70 \mathrm{a}$ & $21,0 \mathrm{~b}$ & 504,96 a & $9,82 \mathrm{a}$ & $3,50 \mathrm{a}$ \\
\hline $50+1 \% \mathrm{CS}$ & 1 & $22,12 \mathrm{~b}$ & $16,42 \mathrm{~b}$ & $19,7 \mathrm{a}$ & $624,91 \mathrm{~b}$ & $12,16 \mathrm{a}$ & $3,77 \mathrm{a}$ \\
\hline $50+.2 \% \mathrm{CS}$ & 1 & $21,76 \mathrm{~b}$ & $16,12 \mathrm{~b}$ & $20,7 \mathrm{~b}$ & $662,39 \mathrm{~b}$ & $15,02 \mathrm{a}$ & $3,68 \mathrm{a}$ \\
\hline $50+0$ & 1 & $23,02 \mathrm{c}$ & $16,79 \mathrm{c}$ & $20,0 \mathrm{~b}$ & $642,43 \mathrm{~b}$ & $13,47 \mathrm{a}$ & $4,31 \mathrm{~b}$ \\
\hline $50+0$ & 2 & $22,78 \mathrm{c}$ & $16,71 \mathrm{c}$ & $19,1 \mathrm{a}$ & $705,44 \mathrm{~b}$ & 13,43 a & $4,24 \mathrm{~b}$ \\
\hline $10+5 \mathrm{TDZ}$ & 1 & $22,40 \mathrm{~b}$ & $16,97 \mathrm{c}$ & $18,5 \mathrm{a}$ & $857,56 \mathrm{c}$ & $18,47 \mathrm{~b}$ & $4,50 \mathrm{~b}$ \\
\hline $10+5 \mathrm{TDZ}$ & 2 & $22,24 \mathrm{~b}$ & $17,29 \mathrm{c}$ & $19,3 \mathrm{a}$ & $898,60 \mathrm{c}$ & $18,82 \mathrm{~b}$ & $4,59 \mathrm{~b}$ \\
\hline CV \% & & 4,73 & 4,38 & 9,77 & 26,55 & 33,80 & 11,79 \\
\hline
\end{tabular}

TABELA 2 - Efeito do ácido giberélico e do tidiazuron sobre a massa fresca dos cachos, dos engaços e das bagas, comprimento e diâmetro das bagas e teor de sólidos solúveis totais da cv. BRS Clara. Jales, 2007.

\begin{tabular}{|c|c|c|c|c|c|c|c|}
\hline \multirow{2}{*}{$\begin{array}{c}\text { Tratamento } \\
\text { GA3 + TDZ } \\
\left(\mathrm{mg} \mathrm{L}^{-1}\right)\end{array}$} & \multirow{2}{*}{$\frac{\mathrm{N}^{\circ} \text { de }}{\text { Aplic. }}$} & \multicolumn{2}{|c|}{ Dimensão de baga (mm) } & \multirow{2}{*}{$\begin{array}{c}\mathrm{SST} \\
\left({ }^{\circ} \mathrm{Brix}\right)\end{array}$} & \multicolumn{3}{|c|}{ Massa fresca $(\mathrm{g})$} \\
\hline & & Comprim. & Diâmetro & & Cacho & Engaço & Bagas \\
\hline Testemunha & 0 & $20,74 \mathrm{a}$ & $15,74 \mathrm{~b}$ & $21,0 \mathrm{e}$ & $355,9 \mathrm{a}$ & $8,62 \mathrm{a}$ & $3,24 \mathrm{a}$ \\
\hline $8+0$ & 4 & $23,78 \mathrm{~d}$ & $16,76 \mathrm{c}$ & $18,2 \mathrm{c}$ & $523,1 \mathrm{c}$ & $12,25 \mathrm{~b}$ & $4,33 \mathrm{~d}$ \\
\hline $8+1$ & 4 & $24,04 \mathrm{~d}$ & $18,15 \mathrm{e}$ & $15,4 \mathrm{a}$ & $601,2 \mathrm{c}$ & $16,68 \mathrm{~d}$ & $4,92 \mathrm{e}$ \\
\hline $10+0$ & 4 & $24,32 \mathrm{~d}$ & $16,37 \mathrm{c}$ & $17,7 \mathrm{c}$ & $434,9 \mathrm{~b}$ & $10,69 \mathrm{~b}$ & $4,30 \mathrm{~d}$ \\
\hline $50+0$ & 2 & $23,68 \mathrm{~d}$ & $15,97 \mathrm{~b}$ & $17,0 \mathrm{~b}$ & $457,8 \mathrm{~b}$ & $11,50 \mathrm{~b}$ & $3,99 \mathrm{c}$ \\
\hline $100+0$ & 1 & $23,20 \mathrm{c}$ & $15,57 \mathrm{~b}$ & $18,5 \mathrm{c}$ & $444,7 \mathrm{~b}$ & $12,00 \mathrm{~b}$ & $3,71 \mathrm{~b}$ \\
\hline $100+0$ & 2 & $24,47 \mathrm{~d}$ & $16,47 \mathrm{c}$ & $16,2 \mathrm{a}$ & $498,9 \mathrm{c}$ & $13,21 \mathrm{c}$ & $4,46 \mathrm{~d}$ \\
\hline $10+5$ & 2 & $22,99 \mathrm{c}$ & $17,47 \mathrm{~d}$ & $17,0 \mathrm{~b}$ & $454,5 \mathrm{~b}$ & $13,88 \mathrm{c}$ & $4,51 \mathrm{~d}$ \\
\hline $10+5$ & 1 & $23,17 \mathrm{c}$ & $17,03 \mathrm{c}$ & $19,7 \mathrm{~d}$ & $506,2 \mathrm{c}$ & $13,48 \mathrm{c}$ & $4,11 \mathrm{c}$ \\
\hline $50+0 *$ & 1 & $22,12 \mathrm{~b}$ & $14,60 \mathrm{a}$ & 20,9 e & $290,3 \mathrm{a}$ & $9,23 \mathrm{a}$ & $3,21 \mathrm{a}$ \\
\hline CV\% & & 5,60 & 4,73 & 9,31 & 29,36 & 32,00 & 12,09 \\
\hline
\end{tabular}

* Tratamento aplicado em conjunto com o raleio de bagas por meio do pente, no período de pré-florção.

\section{CONCLUSÕES}

1- Aplicações parceladas em 2 e 4 vezes de reguladores vegetais melhoram as características do cacho da BRS Clara, provocando aumento no diâmetro, no comprimento e na massa fresca das bagas.

2- Aplicações parceladas de isolada e $\mathrm{GA}_{3}$, ou em mistura com tidiazuron, provocam redução nos teores de sólidos solúveis das bagas da cv. BRS Clara.
3- Baixas dosagens de $\mathrm{GA}_{3}\left(8\right.$ e $10 \mathrm{mg} \mathrm{L}^{-1}$ com ou sem TDZ), em aplicações parceladas, apresentam maior eficiência em relação à aplicação única, em baixas ou altas concentrações.

4- Não é recomendável a utilização do pente na pré-floração para raleio de bagas da cv. BRS Clara. 


\section{REFERÊNCIAS}

BOTELHO, R. V.; PIRES, E. J. P.; TERRA, M. M. Efeitos de reguladores vegetais na qualidade de uvas 'Niagara Rosada’ na região noroeste do Estado de São Paulo. Revista Brasileira de Fruticultura, Jaboticabal, v. 26, n. 1, p. 74-77, 2004.

BOTELHO, R. V.; PIRES, E. J. P.; TERRA, M. M.; CATO, S. C. Efeitos do thidiazuron e do ácido giberélico nas características dos cachos de uva de mesa cultivar Rubi, na região da nova alta paulista. Revista Brasileira de Fruticultura, Jaboticabal, v. 24, n. 2, p. 243-245, 2002.

CAMARGO, U.A.; NACHTIGAL, J.C.; MAIA, J.D.G.; OLIVEIRA, P.R.D.; PROTAS, J.F. da S. BRS Clara: nova cultivar de uva branca de mesa sem semente. Bento Gonçalves: Embrapa Uva e Vinho, 2003. 4p. (Comunicado Técnico, 46).

FEITOSA, C. A. M. Efeitos do CCPU e GA no cultivo de uva-'Itália' na região do submédio São Francisco, Nordeste do Brasil. Revista Brasileira de Fruticultura, Jaboticabal, v. 24, n. 2, p. 348353,2002 .

MIELE, A.; RIZZON, L.A.; DALL'AGNOL, I. Efeito de reguladores de crescimento no tamanho da baga e na composição do mosto da uva 'Itália'. Revista Brasileira de Fruticultura, Jaboticabal, v.22, n.2, p.272-276, 2000.
METIVIER, J. R. Citocininas. In: FERRI, M. G. Fisiologia vegetal 2. São Paulo: EPU, 1986. p. 93-127.

NACHTIGAL, J. C. BRS Clara: recomendações para o cultivo no Rio Grande do Sul. Bento Gonçalves: Embrapa Uva e Vinho, 2007. 8p. (Comunicado Técnico, 74).

NACHTIGAL, J. C.; CAMARGO, U. A.; MAIA, J. D. G. Efeito de reguladores de crescimento em uva apirênica, cv. BRS Clara. Revista Brasileira de Fruticultura, Jaboticabal, v. 27, n. 2, p. 304-307, 2005.

PIRES, E. J. P.; MARTINS, F. P. Técnicas de cultivo. In: POMMER, C.V. (Ed.). Uva: tecnologia de produção, pós-colheita, mercado. Porto Alegre: Cinco Continentes, 2003. p.351-403.

SOUZA LEÃO, P. C. de; MAIA, J. D. G. Aspectos culturais em viticultura tropical - uvas de mesa. Informe Agropecuário, Belo Horizonte, v. 19, n. 194, p. 34-39, 1988.

SOUZA LEÃO, P.C. de; POSSÍDIO, E. L. de. Manejo e tratos culturais. In: SOUZA LEÃO, P. C. de (Ed.). Uva de mesa: produção - aspectos técnicos. Brasília: Embrapa Informação Tecnológica; Petrolina-PE: Embrapa Semiárido, 2001. cap.10, p. 70-81. (Frutas do Brasil, 13).

SOUZA LEÃO, P. C. de; SILVA, D. J.; SILVA, E. E. G. Efeito do ácido giberélico, do bioestimulante crop set e do anelamento na produção e na qualidade da uva 'thompson seedless' no vale do São Francisco. Revista Brasileira de Fruticultura, Jaboticabal, v. 27, n. 3, p. 418-421, 2005 